\title{
Ground-state properties, vortices, and collective excitations in a two-dimensional Bose-Einstein condensate with gravitylike interatomic attraction
}

\author{
A. Keleş, S. Sevinçli, * and B. Tanatar \\ Department of Physics, Bilkent University, 06800 Ankara, Turkey
}

(Received 21 November 2007; published 9 May 2008)

\begin{abstract}
We study the ground-state properties of a Bose-Einstein condensate with short-range repulsion and gravitylike $1 / r$ interatomic attraction in two-dimensions (2D). Using the variational approach we obtain the groundstate energy and analyze the stability of the condensate for a range of interaction strengths in $2 \mathrm{D}$. We also determine the collective excitations at zero temperature using the time-dependent variational method. We analyze the properties of the Thomas-Fermi-gravity and gravity regimes, and we examine the vortex states, finding the coherence length and monopole mode frequency for these regimes. Our results are compared and contrasted with those in $3 \mathrm{D}$ condensates.
\end{abstract}

DOI: 10.1103/PhysRevA.77.053604

PACS number(s): 03.75.Kk, 04.40.-b, 34.20.Cf

\section{INTRODUCTION}

Investigations of Bose-Einstein condensates (BECs) have mostly concentrated on systems with two-body short-ranged interaction which is characterized by the $s$-wave scattering length [1]. Recently, O'Dell et al. [2] proposed a configuration for the occurrence of $1 / r$ interaction which is a totally new regime for cold gases that have a long-range attractive interaction. They showed that a particular spatial configuration of external electromagnetic fields causes a $1 / r$ potential in the near zone. The analysis of this configuration is also important since it suggests a new way to examine the stellar $1 / r$ interaction in the laboratory. Apart from this possibility, it is interesting that such a system results in stable condensates even in the absence of an external trap potential [3]. Recent experiments [4] on polar molecules and condensates with dipole-dipole interactions have started to probe the properties of such systems.

The gravitylike interaction of atoms in a condensate is mostly a result of dipole-dipole interactions [2,5]. By adjustment of the configuration of intense off-resonant laser beams, the $r^{-3}$ term in the dipole-dipole interaction is made to vanish, and one can obtain an interaction of the form $U(\mathbf{r})=-u / \mathbf{r}$ in which $u$ is related to the material parameters and laser intensity. In this system, two new regimes appear where the kinetic energy, contact interaction energy, and gravitylike attractive interaction form a stable configuration without a trap potential [2,5], i.e., the condensate is selfbound. Self-bound condensates have been examined extensively in the literature in the context of laser-induced gravitation [6] and other systems [3]. Ghosh [7] has studied the collective excitation frequencies of this system in threedimensions (3D) within the time-dependent variational method. He showed that variational analysis agrees very well with the results of Ref. [5], in which the sum-rule approach was used. Recently it has been shown that numerical solutions are in very good agreement with variational solutions for 3D systems [8].

There is a growing interest in condensates with longrange interactions, especially in those with dipole-dipole in-

\footnotetext{
*sevilay@fen.bilkent.edu.tr
}

teractions $[9,10]$. After the realization of Bose-Einstein condensation with ${ }^{52} \mathrm{Cr}$ atoms [11], many theoretical and experimental studies on systems with dipolar interaction appeared [12]. The recent progress in the cooling and trapping of neutral atomic gases with an electromagnetic field has also made it possible to study 2D Bose gases [13]. The 2D atomic BECs have many interesting properties as revealed by experiments and theoretical predictions. The excitation spectrum and vortex states of ordinary 2D BECs $[14,15]$ and BECs with dipole-dipole interaction [16] have also been investigated.

In this paper we study a $2 \mathrm{D}$ condensate with attractive $1 / r$ interaction. We calculate the ground-state properties using a variational approach and show that the condensate is stable without the external potential. We also consider the dynamics of the condensate within the time-dependent variational method and calculate the monopole and quadrupole mode frequencies. We examine the excitation spectrum for the Thomas-Fermi-gravity and gravity regimes, analyze the vortex states, and calculate the coherence length as well as the critical angular frequency to create a vortex. Our work parallels a similar consideration for 3D condensates [7] which allows for a comparison of the effects of dimensionality.

\section{GROUND-STATE PROPERTIES AND COLLECTIVE EXCITATIONS}

We will make use of the mean-field theory together with the variational method to investigate the ground-state properties. For a dilute gas of bosonic atoms, we can write the equation of motion for the system,

$$
i \hbar \frac{\partial \psi(\mathbf{r}, t)}{\partial t}=\left(-\frac{\hbar^{2}}{2 m} \nabla^{2}+V_{\text {ext }}(\mathbf{r})+V_{H}(\mathbf{r})\right) \psi(\mathbf{r}, t),
$$

in which $V_{\text {ext }}=m \omega_{0}^{2} r^{2} / 2$ is the external harmonic potential and $V_{H}$ is the Hartree potential, consisting of hard sphere and gravity interactions, respectively, in the form

$$
V_{H}(\mathbf{r})=g|\Psi(\mathbf{r})|^{2}-u \int d^{2} \mathbf{r}^{\prime} \frac{\left|\Psi\left(\mathbf{r}^{\prime}\right)\right|^{2}}{\left|\mathbf{r}^{\prime}-\mathbf{r}\right|},
$$

where $g=2 \sqrt{2 \pi} \hbar^{2} a / m a_{z}$ is the interaction strength with $a$ being the 2D $s$-wave scattering length, and $a_{z}$ is the harmonic 
TABLE I. Comparison of four asymptotic regions.

\begin{tabular}{ccccc}
\hline \hline & $\mathrm{G}$ & TF-G & TF-O & $\mathrm{I}$ \\
\hline \multirow{2}{*}{ Definition } & $\tilde{u} \gg 1$ & $\widetilde{s} \ll \widetilde{u}^{4 / 3}$ & $\tilde{s} \gg 1$ & $\tilde{u} \ll 1$ \\
& $\tilde{s} \ll 1$ & & $\widetilde{s} \gg \widetilde{u}^{4 / 3}$ & $\tilde{s} \ll 1$ \\
$\lambda$ & $1 / \tilde{u}$ & $\widetilde{s} / \widetilde{u}$ & $\widetilde{s}^{1 / 4}$ & 1 \\
$E_{\text {rel }} / \hbar \omega_{0}$ & $\frac{1}{2} N \widetilde{u}^{2}$ & $\frac{1}{2} N \widetilde{u}^{2} \widetilde{s}^{-1}$ & $\frac{1}{2} N \widetilde{s}^{1 / 2}$ & $\frac{1}{2} \mathrm{~N}$ \\
& $\propto N^{3}$ & $\propto N^{2}$ & $\propto N^{3 / 2}$ & $\propto N$ \\
$\rho_{\max }$ & $\frac{N^{3} u^{2}}{16 l_{0}^{4} \hbar^{2} \omega_{0}^{2}}$ & $\frac{N a_{z}^{2} u^{2}}{16 a^{2} l_{0}^{4} \hbar^{2} \omega_{0}^{2}}$ & $\frac{N^{1 / 2} a_{2}^{1 / 2}}{\left(2 \pi^{5}\right)^{1 / 4} a^{1 / 2} l_{0}^{2}}$ & $\frac{N}{\pi l_{0}^{2}}$ \\
\hline \hline
\end{tabular}

oscillator length in the tightly confined direction. We consider a highly anisotropic condensate, i.e., $\omega_{z} \gg \omega_{\perp}$, and use the quasi-three-dimensional scattering model. In this model, if $a_{z} \gg a$, atoms experience collisions in 3D and the contact interaction parameter can be written in terms of the same parameter in 3D [17-19]. One must remember that we can write the Hartree potential in this form if the $-u / r$ potential is sufficiently weak and does not affect the short-range scattering. We use the variational wave function in the form $\Psi(\mathbf{r}, \lambda)=\sqrt{N / \pi \lambda^{2} l_{0}^{2}} \exp \left(-r^{2} / 2 \lambda^{2} l_{0}^{2}\right)$, where $l_{0}=\sqrt{\hbar / m \omega_{0}}$, and this form satisfies the normalization condition with total number of particles $N$. Using this function in the energy functional, the energy per particle can be obtained as

$$
\frac{E(\lambda)}{N \hbar \omega_{0}}=\frac{1}{2}\left(\lambda^{-2}+\lambda^{2}+\tilde{s} \lambda^{-2}-2 \tilde{u} \lambda^{-1}\right),
$$

where we choose the dimensionless interaction parameters as $\tilde{s}=\sqrt{2 / \pi} N a / a_{z}$ and $\tilde{u}=\sqrt{\pi / 2} u N /\left(2 l_{0} \hbar \omega_{0}\right)$. Equation (3) implies that the scattering interaction (third term) in 2D shifts the kinetic energy by $\widetilde{s}$. Minimizing the energy with respect to the variational parameter $\lambda$, we obtain

$$
1-(1+\widetilde{s}) \lambda^{-4}+\widetilde{u} \lambda^{-3}=0 .
$$

One can obtain the virial relation as $-T+V_{\text {ext }}-E_{s}-E_{u} / 2=0$ from a scaling analysis, which is equivalent to the above relation, where $T$ and $V_{\text {ext }}$ are the kinetic and trap potential energies and $E_{s}$ and $E_{u}$ are the interaction energies. It is worth mentioning here that variational calculations have been shown [8] to be quite accurate for a large range of parameters in the case of 3D condensates with gravitylike interaction. Based on our variational results, Table I gives a comparison of the four asymptotic regions on some experimental quantities such as the condensate radius, release energy, and peak density. The radius and release energy are experimentally important quantities since they are the signatures of $1 / r$ interaction [2]. As can be seen from the phase diagram in Fig. 1(a), there are four regions, as in 3D. The ideal noninteracting region (I) and ordinary Thomas-Fermi region (TF-O) are well known from the study of ordinary condensates (with short-range interaction only). The gravity (G) and Thomas-Fermi-gravity (TF-G) regions are related to the balance of the gravitylike potential with the kinetic energy and the contact interaction, respectively. Because these regions are not sensitive to the external potential, it can be adiabatically turned off. The gravitylike attraction does not induce the collapse of the condensate, unlike the contact interaction. Unlike the 3D system [2], a 2D condensate is stable for negative scattering lengths if $\tilde{s}>-1$ irrespective of the value of $\tilde{u}$, whereas there is a sudden collapse for $\tilde{s} \leq$ -1 . In $3 \mathrm{D}$, the condition below which there is no stable condensate becomes $\tilde{s} \tilde{u} \leq 1 / 4$ [2]. From Fig. 1(b), one can conclude that self-bound condensate is stable without the external trap. This can be seen from Eq. (3), which reveals that for small radii gravitylike attraction is always weaker than the kinetic energy; thus the stability of the condensate depends on the balance between the kinetic energy and contact interaction.

We use the time-dependent variational approach to obtain the dynamics of the condensate. The Lagrangian density can be written as [20]

$$
\mathcal{L}=\frac{i \hbar}{2}\left(\psi \frac{\partial \psi^{*}}{\partial t}-\psi^{*} \frac{\partial \psi}{\partial t}\right)-\frac{\hbar^{2}}{2 m}|\nabla \psi|^{2}+\frac{V_{H}(\mathbf{r})}{2}|\psi|^{2},
$$

in which the external potential is set to zero. Oscillation frequencies in 3D obtained by a Gaussian ansatz are compatible with the exact calculations [5]. Thus, we choose the trial function
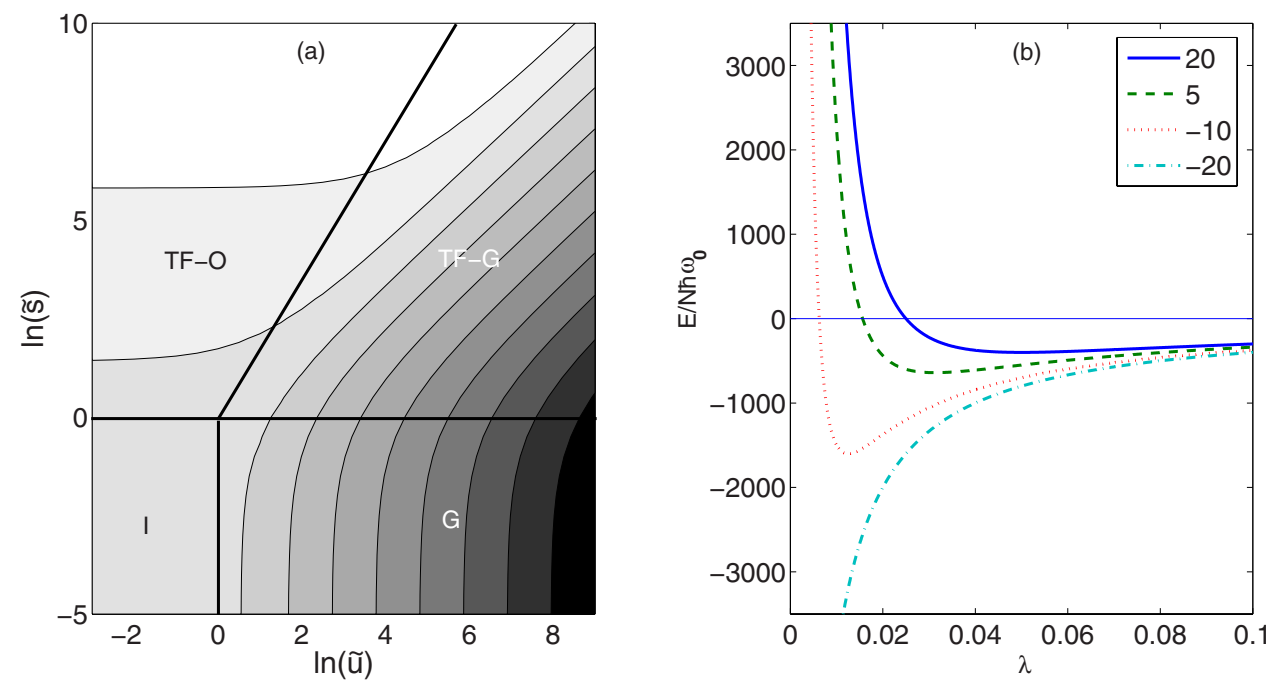

FIG. 1. (Color online) (a) Contour plot of the logarithm of the condensate radius as a function of $\ln \tilde{u}$ and $\ln \tilde{s}$; darker shade corresponds to smaller radius. Four asymptotic regions can be seen from the plot. (b) Ground-state energy of the condensate for different values of the variational parameter $\tilde{s} \tilde{u}$ as a function of $\lambda$, the condensate radius, for large $\tilde{u}$. The energy is scaled by $N \hbar \omega_{0}$ and the radius by $l_{0}$, the harmonic oscillator length. For $\tilde{s} \leq-1$, there is no minimum for finite radius. 


$$
\psi(x, y, t)=\sqrt{\frac{N}{\alpha_{1} \beta_{1} \pi}} \exp \left(-\frac{1}{2}\left[\alpha(t) x^{2}+\beta(t) y^{2}\right]\right),
$$

where the wave function is normalized to $N, x$ and $y$ are variables in units of $l_{0}=\sqrt{\hbar / m \omega_{g}}$, and $\omega_{g}=m u^{2} N^{2} / \hbar^{3}$ is the gravitational frequency. $\alpha(t)=1 / \alpha_{1}^{2}+i \alpha_{2}$ and $\beta(t)=1 / \beta_{1}^{2}$ $+i \beta_{2}$ are the dimensionless time-dependent variational parameters, and $\alpha_{1}$ and $\beta_{1}$ are condensate widths along the $x$ and $y$ directions, respectively. The complex parts of the variational parameters are necessary for an accurate description of the condensate dynamics [21]. Substituting this wave function into the Lagrangian density and integrating over 2D spatial coordinates, we obtain the following Lagrangian:

$$
\begin{aligned}
L= & \frac{S N^{2} u^{2}}{g}\left[\frac{1}{2}\left(\alpha_{1}^{2} \dot{\alpha}_{2}+\beta_{1}^{2} \dot{\beta}_{2}\right)-\frac{1}{2}\left(\frac{1}{\alpha_{1}^{2}}+\alpha_{1}^{2} \alpha_{2}^{2}\right)\right. \\
& -\frac{1}{2}\left(\frac{1}{\beta_{1}^{2}}+\beta_{1}^{2} \beta_{2}^{2}\right)-\frac{1}{\pi} \frac{S}{\alpha_{1} \beta_{1}} \\
& \left.+\sqrt{\frac{\pi}{2}} \frac{{ }_{2} F_{1}\left(\frac{1}{2}, \frac{1}{2} ; 1 ; 1-\beta_{1}^{2} / \alpha_{1}^{2}\right)}{\alpha_{1}}\right],
\end{aligned}
$$

where $S=g m N / 2 \hbar^{2}$ is a dimensionless scattering parameter and ${ }_{2} F_{1}\left(1 / 2,1 / 2 ; 1 ; 1-\beta_{1}^{2} / \alpha_{1}^{2}\right)$ is the hypergeometric function. Note that the scattering parameter $S$ in this part is related to the earlier $\widetilde{s}$ by $S=\pi \widetilde{s}$.

The ground-state energy as a function of the variational parameter in an isotropic system is found to be

$$
E=\frac{S N^{2} u^{2}}{g}\left(\frac{1}{\alpha^{2}}+\frac{1}{\pi} \frac{S}{\alpha^{2}}-\sqrt{\frac{\pi}{2}} \frac{1}{\alpha}\right) .
$$

Minimizing the energy functional with respect to the variational parameter, the equilibrium point is obtained as $\sigma$ $=(2 / \pi)^{1 / 2}(2+2 S / \pi)$. The chemical potential $\mu=\partial E / \partial N$ and sound velocity $c_{s}^{2}=\mu / m$ can be calculated from Eq. (8). Using the Euler-Lagrange equations, the time evolutions of the widths are

$$
\begin{aligned}
& \ddot{\alpha}_{1}=\frac{1}{\alpha_{1}^{3}}+\sqrt{\frac{\pi}{2}}\left(\frac{\tilde{S}}{\alpha_{1}^{2} \beta_{1}}+F_{\alpha_{1}}\right), \\
& \ddot{\beta}_{1}=\frac{1}{\beta_{1}^{3}}+\sqrt{\frac{\pi}{2}}\left(\frac{\tilde{S}}{\alpha_{1} \beta_{1}^{2}}+F_{\beta_{1}}\right),
\end{aligned}
$$

where $\tilde{S}=\sqrt{2 / \pi^{3}} S$, and $F_{\alpha_{1}}$ and $F_{\beta_{1}}$ are the derivatives of ${ }_{2} F_{1}\left(1 / 2,1 / 2 ; 1 ; 1-\beta_{1}^{2} / \alpha_{1}^{2}\right) / \alpha_{1}$ with respect to $\alpha_{1}$ and $\beta_{1}$, respectively. We are looking for low-energy excitations which correspond to small oscillations around the equilibrium point. Thus, we perform an expansion around the equilibrium width by letting $\alpha_{1}=\sigma+\delta \alpha_{1}$ and $\beta_{1}=\sigma+\delta \beta_{1}$ for the isotropic system. The time evolution of the widths is given by

$$
\begin{aligned}
\delta \ddot{\alpha}_{1}= & {\left[-\frac{3}{\sigma^{4}}+\sqrt{\frac{\pi}{2}}\left(-\frac{2 \tilde{S}}{\sigma^{4}}+\frac{5}{8 \sigma^{3}}\right)\right] \delta \alpha_{1} } \\
& +\sqrt{\frac{\pi}{2}}\left(-\frac{S}{\sigma^{4}}+\frac{3}{8 \sigma^{3}}\right) \delta \beta_{1},
\end{aligned}
$$

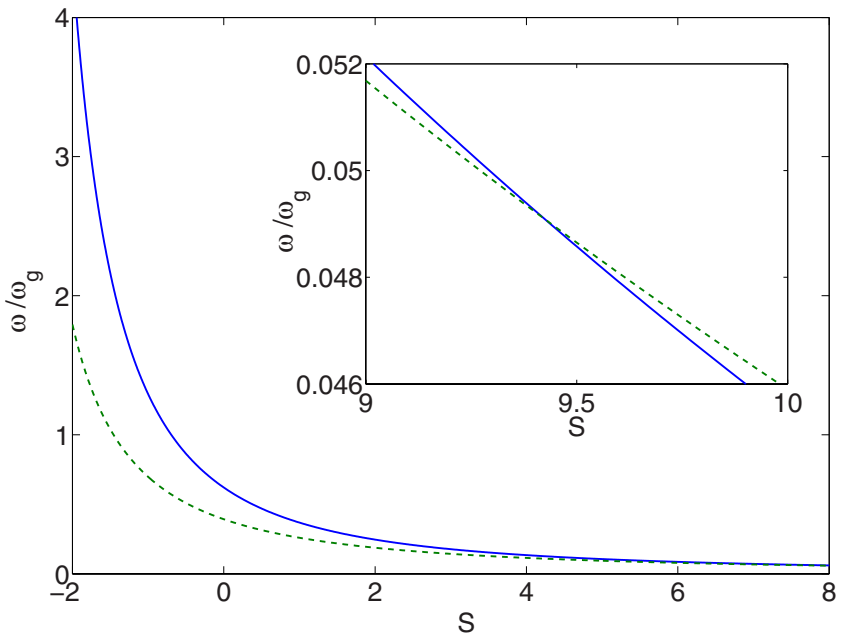

FIG. 2. (Color online) Monopole (dashed line) and quadrupole mode (solid line) frequencies $\left(\omega_{M}\right.$ and $\omega_{Q}$, respectively) as functions of the dimensionless scattering parameter $S$. Inset shows the intersection of two modes.

$$
\begin{aligned}
\delta \ddot{\beta}_{1}= & \sqrt{\frac{\pi}{2}}\left(-\frac{\tilde{S}}{\sigma^{4}}+\frac{3}{8 \sigma^{3}}\right) \delta \alpha_{1} \\
& +\left[-\frac{3}{\sigma^{4}}+\sqrt{\frac{\pi}{2}}\left(-\frac{2 \tilde{S}}{\sigma^{4}}+\frac{5}{8 \sigma^{3}}\right)\right] \delta \beta_{1} .
\end{aligned}
$$

Substituting $e^{i \omega t}$-type solutions in the above set of equations, we obtain the following excitation frequencies:

$$
\begin{aligned}
& \omega_{+}^{2}=\frac{3}{\sigma^{4}}+\sqrt{\frac{\pi}{2}}\left(\frac{3 \tilde{S}}{\sigma^{4}}-\frac{1}{\sigma^{3}}\right), \\
& \omega_{-}^{2}=\frac{3}{\sigma^{4}}+\sqrt{\frac{\pi}{2}}\left(\frac{\tilde{S}}{\sigma^{4}}-\frac{1}{4 \sigma^{3}}\right) .
\end{aligned}
$$

The excitation spectrum for a 2D condensate with gravitylike interaction is plotted in Fig. 2 as a function of the dimensionless scattering parameter. We observe that, in contrast to $3 \mathrm{D}$, the $2 \mathrm{D}$ system can bear the negative scattering parameter down to $S=-\pi$. For large values of the scattering parameter, the pseudopotential term dominates the gravitational energy and the monopole mode is more energetic than the quadrupole mode. At $S=9.42$, there is an intersection of the two modes which can be seen from the inset of Fig. 2, where it is not possible to distinguish two modes experimentally. A similar crossing occurs in 3D at a larger value of $S$ [7].

$T F-G$ regime. When the gravitylike potential is balanced by the contact interaction i.e., for large $s$-wave scattering lengths, the kinetic energy can be neglected. The total energy of the ground state becomes $E_{0}=-0.62\left(N^{2} u^{2} / g\right)$. The ground-state energy per particle varies with $N$ as in 3D. In this regime, monopole and quadrupole frequencies are obtained as $\omega_{Q}=1.5462 \omega_{g} S^{-3 / 2}$ and $\omega_{M}=2.1867 \omega_{g} S^{-3 / 2}$. Their ratio is $\omega_{M} / \omega_{Q}=1.42$, which is 1.58 in $3 \mathrm{D}$. Note also that the dependence of $\omega_{Q}$ and $\omega_{M}$ on $S$ in $3 \mathrm{D}$ is $\sim S^{-3 / 4}$, which is a distinctive feature. The facility with which the $s$-wave scattering length can be tuned through the Feshbach resonance 
makes the results of our calculation amenable to experimental investigations. The identification of the $2 \mathrm{D}$ nature of condensates is thus possible from the study of collective excitations.

$G$ regime. In this regime we neglect the contact interaction; this is the analog of the nonrelativistic boson star [22]. The ground-state energy per particle varies with $N$ similarly to the case in the TF-G regime. Quadrupole and monopole mode frequencies are calculated as $\omega_{Q}=0.6269 \omega_{g}$ and $\omega_{M}$ $=0.3927 \omega_{g}$, respectively, in terms of the gravitational frequency $\omega_{g}$. Their ratio is $\omega_{M} / \omega_{Q}=0.63$ in this regime, which is to be compared with 0.60 in $3 \mathrm{D}$.

We now discuss the experimental feasibility of $2 \mathrm{D}$ condensates with $1 / r$ interaction against losses. The main sources of depletion in a gravitylike interacting gas, in the TF-G and G regions, are losses due to $1 / r^{3}$ oscillating interfering terms and those due to incoherent phonon scattering, namely, Rayleigh scattering. The conditions necessary to observe the transition from external trapping to self-binding have been analyzed by O'Dell et al. [2] and Giovanazzi et al. [6] in the case of 3D condensates. They have shown that the Rayleigh scattering rate is reduced by a factor $\left(q R_{\mathrm{rms}}\right)^{2}$ for sample sizes smaller than the laser wavelength $[2,6]$. Here $q$ is the wave vector associated with the laser wavelength (i.e., $\left.q=2 \pi / \lambda_{L}\right)$ and $R_{\mathrm{rms}}$ is the root mean square of the condensate size proportional to the variational width parameter. Our numerical calculations for the TF-G region give $R_{\text {rms }}$ $\sim 0.46 \lambda_{L}$ for the values $a \sim 3 \mathrm{~nm}, a_{*} \sim 10 \mathrm{~cm}$, and $\lambda_{L}$ $=10.6 \mu \mathrm{m}$, which means that this region is experimentally accessible.

We calculate the temporal characteristics in relation to Rayleigh scattering rate by adopting the approach of Giovanazzi et al. [6] to a $2 \mathrm{D}$ condensate. In the TF-G and G regimes, the characteristic time scale for the dynamics of the system can be estimated from the plasma frequency, which has the form

$$
\omega_{p}^{2}=\frac{4 \pi^{2} u \rho_{\text {peak }}}{m \lambda_{L}}
$$

in 2D [23], where $\rho_{\text {peak }}$ is the peak density and $\lambda_{L}$ is the laser wavelength. The Rayleigh scattering rate can be expressed as [6]

$$
\Gamma_{\text {Ray }}=\frac{20 \pi}{11} \frac{u}{\hbar \lambda_{L}} .
$$

Using Eq. (16) and recoil energy $E_{R}=\hbar^{2} q^{2} / 2 m$, one can write the plasma frequency $\omega_{p}$ as

$$
\omega_{p}=0.72 \frac{\Gamma_{\mathrm{Ray}}^{3 / 2} N^{3 / 2}}{\left(E_{R} / \hbar\right)^{1 / 2}} \frac{1}{1+\widetilde{s}} .
$$

For a $2 \mathrm{D}$ condensate, $a_{z} \gg a$ and the last quotient in the above expression goes to unity since $\tilde{s} \rightarrow 0$. Thus, for the parameters given in Ref. [6], namely, $N=40$ atoms, $\Gamma_{\text {Ray }}=1.58$ $\times 10^{4} \mathrm{~s}^{-1}$, and recoil energy $E_{R} / \hbar=1.57 \times 10^{5} \mathrm{~s}^{-1}$, we find $\omega_{p} \approx 57 \Gamma_{\text {Ray }}$, which is about three times the value in the $3 \mathrm{D}$ case [23]. This estimation shows that even for a small number of atoms the proposed laser characteristics and twodimensional nature of the condensate allow for several oscil- lations of the self-bound gas within the Rayleigh lifetime.

One may also compare the monopole and quadrupole mode frequencies in the TF-G regime to the Rayleigh scattering rate. Recalling, for instance, that $\omega_{Q}=1.5462 \omega_{g} S^{-3 / 2}$, the ratio of the TF-G quadrupole mode to $\Gamma_{\text {Ray }}$ is

$$
\frac{\omega_{Q}}{\Gamma_{\text {Ray }}}=0.94 \frac{\hbar \Gamma_{\text {Ray }} N^{2} S^{-3 / 2}}{E_{R}} .
$$

For the parameters given above, we find that $\omega_{Q} / \Gamma_{\text {Ray }}<53$ for $S<2$ and $\omega_{Q} / \Gamma_{\text {Ray }}<1$ for $S>28$. Our estimation again shows that excitations can be observed experimentally within the Rayleigh lifetime.

\section{VORTICES}

Vortices in Bose-Einstein condensed systems are important as they experimentally reveal the macroscopic phase coherence properties. To study the vortex states we again use the time-dependent variational analysis and choose the variational wave function for the self-bound gas as

$$
\begin{aligned}
\psi(\mathbf{r}, t)= & \sqrt{\frac{N}{\pi q ! \alpha^{2 q+2}}} r^{q} \exp \left(-\frac{r^{2}}{2}\left[1 / \alpha(t)^{2}\right.\right. \\
& +i \beta(t)]) \exp (i q \phi),
\end{aligned}
$$

where $q$ is the vortex quantum number. By using the same Lagrangian density in Eq. (5), we obtain the Lagrangian

$$
\begin{aligned}
L= & \frac{S N^{2} u^{2}}{g}\left[(q+1) \alpha^{2} \dot{\beta}-(q+1)\left(\frac{1}{\alpha^{2}}+\alpha^{2} \beta^{2}\right)\right. \\
& \left.-\frac{g_{q}}{\pi} \frac{S}{\alpha^{2}}+\sqrt{\frac{\pi}{2}} \frac{c_{q}}{\alpha}\right],
\end{aligned}
$$

where $g_{q}=(2 q) ! / 2^{2 q}(q !)^{2}, \quad c_{1}=7 / 16$, and $c_{2}=321 / 1024$. Then, one can find the energy of the vortex state as

$$
E_{q}=\frac{S N^{2} u^{2}}{g}\left((q+1) \frac{1}{\alpha^{2}}+\frac{g_{q}}{\pi} \frac{S}{\alpha^{2}}-\sqrt{\frac{\pi}{2}} \frac{c_{q}}{\alpha}\right) .
$$

The equilibrium point is found by minimizing the energy

$$
\sigma_{q}=\sqrt{\frac{2}{\pi}}\left(\frac{2(q+1)+(2 / \pi) g_{q} S}{c_{q}}\right) .
$$

The balance between the kinetic energy and the interaction energy terms fixes the structure of the vortex core. The coherence length $\xi$ is a measure of the superfluid characteristics of the system, which is obtained by equating these three energies:

$$
\frac{\hbar^{2}}{2 m \xi}=\frac{g N}{2 R^{2}}-\frac{u N}{\xi},
$$

where $R=g \sqrt{F} / 2 u S$ is the radius of the condensed state and $F=-\left(\frac{1}{\sigma^{2}}+\frac{S}{\pi \sigma^{2}}-\sqrt{\frac{\pi}{2}} \frac{1}{\sigma}\right)$, in which $\sigma$ is the equilibrium width without vortices. Thus, the coherence length is 


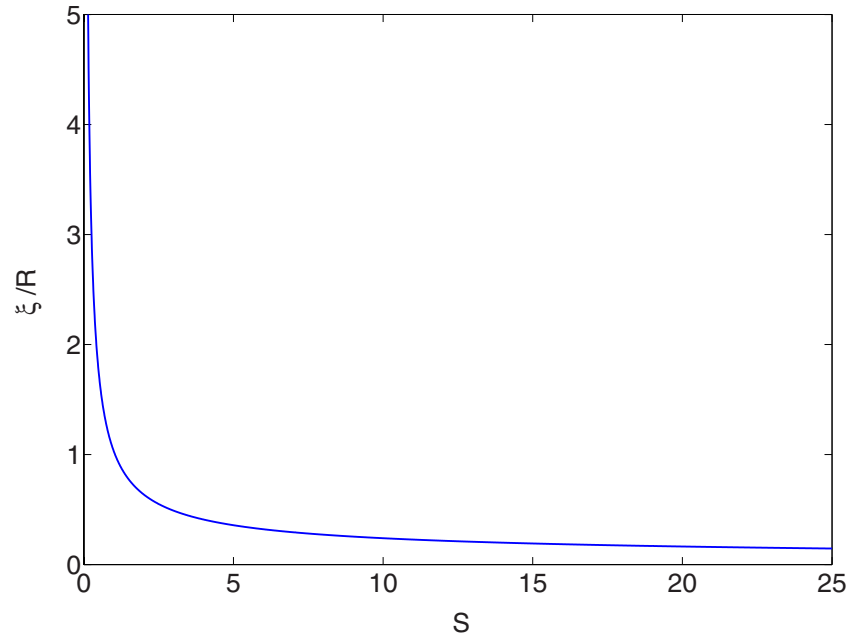

FIG. 3. (Color online) Coherence length as a function of the dimensionless scattering parameter $S$.

$$
\frac{\xi}{R}=\frac{\sqrt{F}+\sqrt{F+2 S}}{2 S} .
$$

The coherence length as a function of the scattering parameter $S$ is shown in Fig. 3. For small values of the scattering parameter, $\xi / R$ is seen to be very large, which implies that superfluidity is destroyed. As the scattering length increases, the coherence length is getting much smaller, i.e., the system keeps displaying superfluidity.

The critical angular frequency to create a vortex is found by using the energies of the vortex and vortex-free states as

$\Omega_{q}=\frac{\omega_{g}}{2}\left[\frac{2}{\sigma_{q}^{2}}-\frac{1}{\sigma^{2}}+\frac{S}{2 \pi}\left(\frac{1}{2 \sigma_{q}^{2}}-\frac{1}{2 \sigma^{2}}\right)-\sqrt{\frac{\pi}{2}}\left(\frac{7}{16 \sigma_{q}}-\frac{1}{\sigma}\right)\right]$.

In Fig. 4, we present the behavior of the critical angular frequency for one vortex. From the inset one can see that $\Omega_{1}$ increases for negative $S$, i.e., for attractive interaction it is

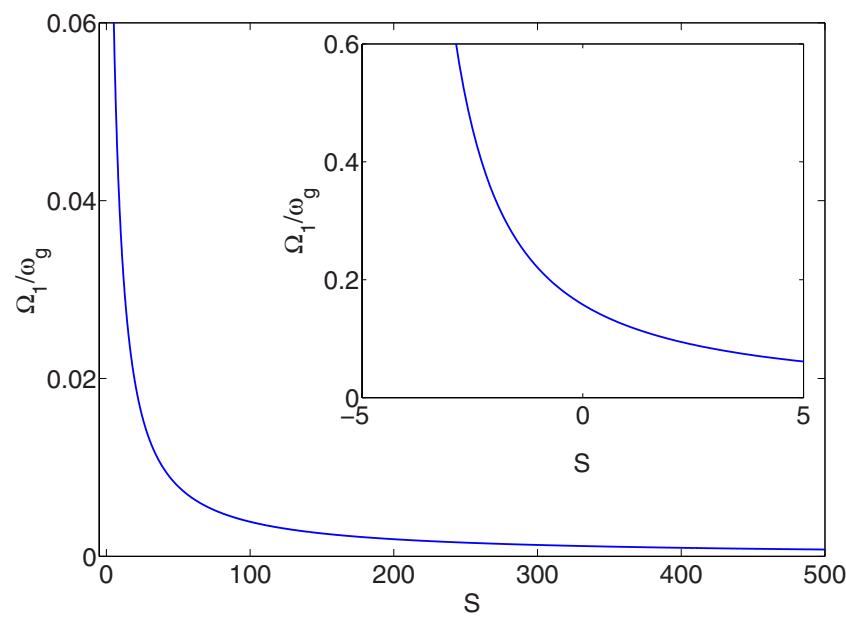

FIG. 4. (Color online) Critical angular frequency for $q=1$ as a function of the dimensionless scattering parameter $S$. Inset is a zoom plot for negative $S$ values. harder to create a vortex, and it decreases as the scattering parameter increases.

The frequency of the monopole mode for the vortex state is obtained as

$$
\omega_{q}^{2}=\left(\frac{3}{\sigma_{q}^{4}}+\frac{3 g_{q} S}{\pi(q+1) \sigma_{q}^{4}}-\sqrt{\frac{\pi}{2}} \frac{c_{q}}{(q+1) \sigma_{q}^{3}}\right),
$$

where $\sigma_{q}$ is defined in Eq. (22). In the presence of vortices, the system collapses at $S=-(q+1) \pi / g_{q}$.

$T F-G$ regime. When the $s$-wave scattering length is very large, the kinetic energy contribution can be neglected. Using Eq. (24), the superfluid coherence length is obtained as $\xi / R=0.7071 S^{-1 / 2}$ where $F=1.23 S^{-1}$. As $S$ increases, the coherence length gets smaller compared to the size of the condensate, i.e., superfluid properties are observed in the TF-G regime. The critical angular frequencies needed to create vortices are $\Omega_{1}=0.3808 \omega_{g} S^{-1}$ and $\Omega_{2}=0.2277 \omega_{g} S^{-1}$ for $q=1$ and 2 , respectively. Unlike in the $3 \mathrm{D}$ case, these two frequencies are larger than the chemical potentials $\mu_{1}$ and $\mu_{2}$; thus the condensate state with a vortex is unbounded in this regime.

$G$ regime. In this regime the $s$-wave interaction energy is very small, so it is neglected. The radius of the condensate is $R_{g}=g \sqrt{F} / 2 u S$ and the superfluid coherence length is $\xi$ $=0.798 R_{g}$, close to the radius of the condensate, which means that superfluidity disappears. This ratio is larger in the 3D system [7]. The critical angular frequencies are $\Omega_{1}$ $=0.1776 \omega_{g}$ and $\Omega_{2}=0.095 \omega_{g}$ for $q=1$ and 2 , respectively, and $\Omega_{2}$ is much less than $\Omega_{1}$. The chemical potentials in the rotating frame are $\left|\mu_{1}\right| / \hbar=0.0188 \omega_{g}$ and $\left|\mu_{2}\right| / \hbar=0.0065 \omega_{g}$. In this regime, only one monopole mode is stable: $\omega_{1}$ $=0.0188 \omega_{g}$. For $q=2$, the monopole mode frequency $\omega_{2}$ $=0.0096 \omega_{g}>-\mu / \hbar$, which means that this oscillation is unstable and particles can escape from the condensate.

A recent paper by Giovanazzi, Santos, and Pfau [12] discusses the way in which accurate determination of the $s$-wave scattering length can be made using the collective oscillation frequencies in a dipolar BEC. We surmise that a similar idea may be developed in the present context of gravitylike $1 / r$ interaction.

\section{CONCLUSIONS}

In this paper, we have shown that a laser-induced attractive $1 / r$ interaction gives rise to a stable condensate in 2D without a trap as in the $3 \mathrm{D}$ case. Unlike the $3 \mathrm{D}$ case, there is no collapse till $\tilde{s}=-1$. We have calculated experimental quantities such as the release energy, the peak velocity, and the condensate radius for the I, TF-O, TF-G, and $\mathrm{G}$ regions. We have also studied the dynamics of the system and calculated the monopole and quadrupole frequencies and analyzed them within the TF-G and $\mathrm{G}$ regimes. These modes depend on the scattering length $a$ in the TF-G regime, unlike the case in the ordinary TF regime. We have shown that the monopole mode exists not only for positive $S$ values but also for negative values down to $S=-\pi$, in contrast to the situation in $3 \mathrm{D}$ [7] where the monopole mode exists for $S>0$ only. Our estimate of the main loss mechanism, namely, the Rayleigh 
scattering, shows that these collective oscillations may be observed in 2D condensates for reasonable parameters.

We have also investigated the vortex states and calculated the energy, the coherence length, the critical angular frequencies, and the monopole mode frequencies for $q=1,2$. The presence of vortices in the condensate extends the range of $S$ in which the condensate is stable. As the scattering parameter increases, the system keeps displaying superfluidity since the coherence length is getting smaller. We have examined the TF-G and $G$ regimes and have shown that in the TF-G regime the condensate state is unbounded. In the $G$ regime, superfluidity disappears and only the monopole mode for the vortex state for $q=1$ is stable.

It would be interesting to study the collective excitations of the present system within the sum-rule approach [5].
Comparison of the time-dependent variational method and the sum-rule approach has yielded a very good agreement in 3D condensates with gravitylike attraction. A similar comparison would provide a further assessment of the reliability of our results. Finally, the results of our calculations should be useful in analyzing experiments performed on 2D condensates. The distinctive features of collective modes may help in the identification of the $2 \mathrm{D}$ nature of condensates in various regimes compared to the $3 \mathrm{D}$ case.

\section{ACKNOWLEDGMENTS}

This work is supported by TUBITAK (Grant No. 106T052) and TUBA. We thank M. Ö. Oktel for fruitful discussions.
[1] F. Dalfovo, S. Giorgini, L. P. Pitaevskii, and S. Stringari, Rev. Mod. Phys. 71, 463 (1999).

[2] D. O'Dell, S. Giovanazzi, G. Kurizki, and V. M. Akulin, Phys. Rev. Lett. 84, 5687 (2000).

[3] H. Saito and M. Ueda, Phys. Rev. Lett. 90, 040403 (2003); P. G. Kevrekidis, G. Theocharis, D. J. Frantzeskakis, and B. A. Malomed, ibid. 90, 230401 (2003); G. D. Montesinos, V. M. Perez-Garcia, and H. Michinel, ibid. 92, 133901 (2004); M. Matuszewski, E. Infeld, B. A. Malomed, and M. Trippenbach, ibid. 95, 050403 (2005).

[4] J. Stuhler, A. Griesmaier, T. Koch, M. Fattori, T. Pfau, S. Giovanazzi, P. Pedri, and L. Santos, Phys. Rev. Lett. 95, 150406 (2005).

[5] S. Giovanazzi, G. Kurizki, I. E. Mazets, and S. Stringari, Europhys. Lett. 56, 1 (2001).

[6] S. Giovanazzi, D. O’Dell, and G. Kurizki, Phys. Rev. A 63, 031603(R) (2001).

[7] T. K. Ghosh, Phys. Rev. A 65, 053616 (2002).

[8] I. Papadopoulos, P. Wagner, G. Wunner, and J. Main, Phys. Rev. A 76, 053604 (2007).

[9] K. Goral and L. Santos, Phys. Rev. A 66, 023613 (2002).

[10] L. Santos, G. V. Shlyapnikov, and M. Lewenstein, Phys. Rev.
Lett. 90, 250403 (2003).

[11] A. Griesmaier, J. Werner, S. Hensler, J. Stuhler, and T. Pfau, Phys. Rev. Lett. 94, 160401 (2005).

[12] S. Giovanazzi, L. Santos, and T. Pfau, Phys. Rev. A 75, 015604 (2007).

[13] A. Posazhennikova, Rev. Mod. Phys. 78, 1111 (2006).

[14] L. Pricoupenko, Phys. Rev. A 70, 013601 (2004).

[15] L. D. Carr and C. W. Clark, Phys. Rev. Lett. 97, 010403 (2006).

[16] U. R. Fischer, Phys. Rev. A 73, 031602(R) (2006).

[17] M. D. Lee, S. A. Morgan, M. J. Davis, and K. Burnett, Phys. Rev. A 65, 043617 (2002).

[18] L. Salasnich, A. Parola, and L. Reatto, Phys. Rev. A 65, 043614 (2002).

[19] B. Tanatar, A. Minguzzi, P. Vignolo, and M. P. Tosi, Phys. Lett. A 302, 131 (2002).

[20] L. Salasnich, Int. J. Mod. Phys. B 14, 1 (2000).

[21] V. M. Perez-Garcia, H. Michinel, J. I. Cirac, M. Lewenstein, and P. Zoller, Phys. Rev. Lett. 77, 5320 (1996).

[22] X. Z. Wang, Phys. Rev. D 64, 124009 (2001).

[23] C. I. Um, W. H. Kahng, E. S. Yim, and T. F. George, Phys. Rev. B 41, 259 (1990). 